significant parametric pairwise t-test $(\mathrm{p} \leq 0.05)$ and ii) area under the receiver-operator characteristic curve (AUROC $\geq 0.59$ ) were selected for inclusion in a logistic regression model.

Results The biomarkers that met criteria and were taken forward for further analysis are shown in Table 1. Of these CC16, CRP, MMP8 and NGAL combined had an AUROC 0.82 (95\% confidence interval 0.74 to 0.90$)$. The Youden's index gave a sensitivity and specificity of $78 \%$ and $82 \%$ respectively.

Conclusion COPD exacerbations can be identified by urinary biomarkers. The biomarker panel requires further validation in a prospective longitudinal study.

\begin{tabular}{lcl}
$\begin{array}{l}\text { Abstract P185 Table } 1 \\
\text { exacerbation short listed from a list of 50 biomarkers }\end{array}$ & \\
\hline Biomarker & AUROC & $\begin{array}{l}\text { p- } \\
\text { value }\end{array}$ \\
\hline Fibrinogen & 0.6395 & 0.0029 \\
Secretory leukocyte peptidase inhibitor (SLPI) & 0.5917 & 0.0268 \\
Interleukin - 6 (IL-6) & 0.6025 & 0.0325 \\
N-Formyl-methionyl-leucyl-phenylalanine (fMLP) & 0.6132 & 0.0121 \\
Desmosine & 0.6210 & 0.0386 \\
Clara Cell protein (CC16) & 0.6405 & 0.0025 \\
Tissue Inhibitor of Metalloproteinase 1 (TIMP 1) & 0.6446 & 0.0325 \\
Tissue Inhibitor of Metalloproteinase 2 (TIMP 2) & 0.6271 & 0.0287 \\
C-reactive Protein(CRP) & 0.6463 & 0.0002 \\
Chitinase 3 like 1 protein (CH3L1) & 0.6172 & 0.0139 \\
Alpha 1 Anti-Trypsin (A1AT) & 0.7240 & $6.3 \mathrm{E}-6$ \\
Creatinine & 0.6460 & 0.0009 \\
Beta -2 Microglobulin (B2M) & 0.6972 & 0.0001 \\
Cystatin C & 0.6747 & 0.0098 \\
Matrix Metalloproteinase 8 (MMP8) & 0.6003 & 0.0170 \\
Neutrophil Gelatinase-Associated Lipocalin & 0.6169 & 0.0072 \\
(NGAL) & & \\
\hline
\end{tabular}

\section{P186 DEMONSTRATING HIGHER DIFFUSION COEFFICIENTS IN PATIENTS WITH EOSINOPHILIC VS. NON-EOSINOPHILIC EXACERBATIONS OF COPD}

${ }^{1} \mathrm{R}$ Barker, ${ }^{2} \mathrm{C}$ Jones, ${ }^{2} \mathrm{~J}$ Fenton-Woods, ${ }^{2} \mathrm{~L}$ Smith, ${ }^{2} \mathrm{M}$ Johnson, ${ }^{2} \mathrm{R}$ Barker. ${ }^{2}$ University of Oxford, Oxford, UK; ${ }^{2}$ Kings College Hospital, London, UK

\subsection{6/thoraxjnl-2017-210983.328}

Background There is evidence that acute exacerbations of COPD (AECOPD) may be stratified by peripheral blood eosinophil count and that this may allow identification of a pathologically distinct phenotype with clinical value. We have compared lung function measures between patients with eosinophilic and non-eosinophilic AECOPD.

Methods All admissions to an inner London teaching hospital with AECOPD were recorded between 2004 and 2012. The eosinophilic phenotype is defined as a first blood count within 24 hours of admission showing peripheral blood eosinophil count $32 \%$ of white blood cells. The first lung function test recorded, performed in a dedicated-laboratory between admissions, were used in analysis. We used non-parametric statistics (Mann-Witney U) for univariate comparisons, and linear regression for multivariate analyses.
Results There were 2793 admissions with AECOPD recorded, we used only first admissions $(1,279)$. Of the first admissions $1104(86.3 \%)$ have a recorded peripheral blood eosinophil phenotype $(32=331,<2=773)$ and 821 (64.1\%) had a recorded FEV1. Of 821 the mean age was 69, 364 were female and 457 male. Proportion with eosinophilic AECOPD 31.1\%. The mean FEV1 was 1.12 L (SD 0.55), mean FEV1\% predicted 45.47 (SD 20.46) and Mean FEV1/VC (47.78 SD 15.88) The eosinophilic group had a higher FEV1 (mean 1.26 vs $1.05, \mathrm{p}<0.001$ ) and higher percent predicted FEV1 (mean 48.75 vs. $44.00, p=0.01$ ) than those in the non-eosinophilic group. 565 patients had $\mathrm{D}_{\mathrm{L}} \mathrm{CO}$ measured (44.2\%). Eosinophilic phenotype was associated with a higher $\mathrm{D}_{\mathrm{L}} \mathrm{CO}$ even after adjusting for FEV1\% predicted $(\mathrm{p}<0.001)$.

Figure 1 - A comparison of $\mathrm{D}_{\mathrm{L}} \mathrm{CO}$ percent predicted measured in stable state, in patients with eosinophilic vs. non-eosinophilic AECOPD admissions.

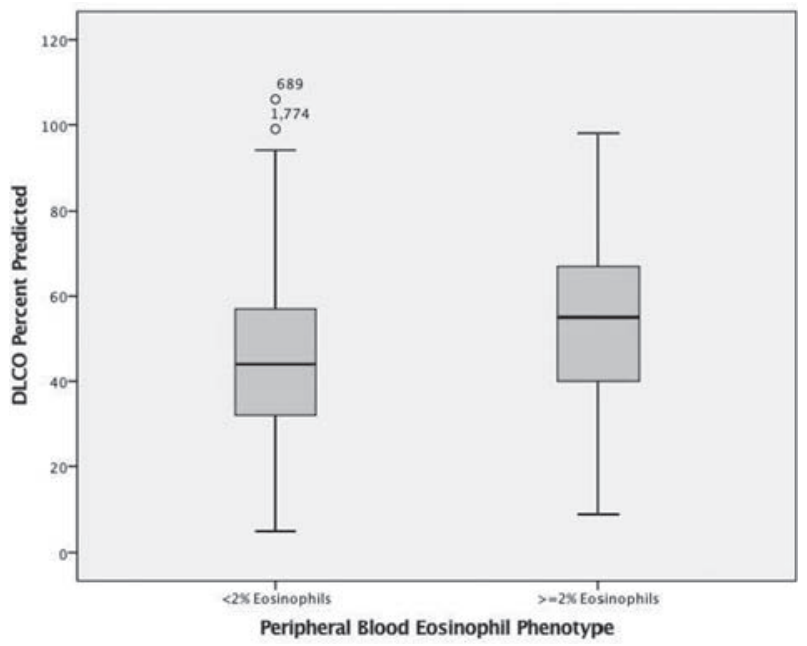

Abstract P186 Figure 1

Discussion In this cohort, patients admitted to hospital with acute exacerbations of COPD associated with an eosinophilic phenotype had a higher FEV1 and higher DLCO\% predicted. A lower DLCO is more associated with an "emphysematous" than "bronchial" form of COPD. Our Results suggest that the eosinophilic phenotype of AECOPD may be associated with less alveolar and pulmonary capillary damage.

\section{P187 SEASONALITY OF EOSINOPHILIC AND NON- EOSINOPHILIC EXACERBATIONS OF COPD}

${ }^{1,2} \mathrm{R}$ Barker, ${ }^{1} \mathrm{R}$ Shrimanker, ${ }^{1} \mathrm{R}$ Russell, ${ }^{2} \mathrm{~J}$ Fenton-Woods, ${ }^{2} \mathrm{C}$ Jones, ${ }^{2} \mathrm{~L}$ Smith, ${ }^{2} \mathrm{M}$ Johnson, ${ }^{1}$ I Pavord. 'Oxford University, Oxford, UK; ${ }^{2}$ King's College Hospital, London, UK

\subsection{6/thoraxjnl-2017-210983.329}

Background Patients presenting with an acute exacerbation of COPD (AECOPD) and a peripheral blood eosinophil count $\geq=2 \%$ of the total white cell count have a better response to oral cortico-steroids, suggesting that stratification by this biomarker identifies a pathologically distinct phenotype 